\title{
Trace element complexation by humic substances: issues related to quality assurance
}

\author{
Montserrat Filella $\cdot$ Wolfgang Hummel
}

Received: 22 April 2010/Accepted: 7 October 2010/Published online: 23 October 2010

(C) Springer-Verlag 2010

\begin{abstract}
Despite the amount of data available and the effort that has been put into studying the binding of trace elements by humic substances, there is still a significant amount of uncertainty surrounding the validity and applicability of the existing values in solving particular problems in the environmental, toxicity and industrial fields. This paper discusses the problems associated with the characterisation of humic binding and proposes a general approach for quality assessing trace element-humic substances complexation data, which includes the normalisation of raw experimental data prior to any data treatment (modelling).
\end{abstract}

Keywords Humic substances $\cdot$ Metal binding $\cdot$ Metal complexation · Quality assurance $\cdot$ Aquatic chemistry

\section{Introduction}

A significant proportion of the natural organic matter (NOM) present in different environmental compartments is composed of fairly stable compounds produced either in soils or in water bodies [1]. Because of their resistance to

This article is part of the Topical Issue "Quality Assurance of Thermodynamic Data".

\section{Filella (凹)}

Institute F.-A. Forel, University of Geneva,

10 route de Suisse, 1290 Versoix, Switzerland

e-mail: montserrat.filella@unige.ch

M. Filella

SCHEMA, 92 rue Principale, 6990 Rameldange, Luxembourg

W. Hummel

Laboratory for Waste Management, Paul Scherrer Institute,

5232 Villigen, Switzerland degradation, these compounds are sometimes called refractory organic matter (ROM) but they are most often referred to as humic acids or humic substances. According to the International Humic Substances Society (IHSS), "humic substances are complex and heterogeneous mixtures of polydispersed materials formed in soils, sediments and natural waters by biochemical and chemical reactions during the decay and transformation of plant and microbial remains" [2]. Historically, this name stems from the widespread practice in soil science of isolating two fractions of NOM, the so-called humic and fulvic acids, on the basis of their solubility in concentrated acid and base solutions [3]. Humic substances have attracted the attention of scientists from a wide variety of disciplines because they play a part in numerous processes in soils and natural waters: e.g. soil weathering, plant nutrition, $\mathrm{pH}$ buffering, mobility, bioavailability and toxicity of trace metals, degradation and transport of hydrophobic organic chemicals, formation of disinfection by-products during water treatment, etc. In this article, no distinction will be made between fulvic and humic acids (except in the few cases when differences in their composition are mentioned) and only the terms 'humics' and 'humic substances', which include both, will be used.

It is currently accepted - and repeated in the introduction of many articles and books - that humics are largely responsible for the complexation of trace elements by NOM in waters and soils (e.g. they are "the most chemically significant fraction of NOM" [4] or are "widely believed to be representatives of NOM behaviour" [5]). For this reason, the study of trace element complexation by humic substances has been the focus of considerable research and an impressive amount of literature exists on the topic (e.g. a comprehensive compilation currently contains around 700 articles [6]), with the earliest studies 
dating back to the late 1950 s (e.g. $[7,8]$ ). Whether the role played by humic substances is as decisive and generalised as has often claimed probably merits further discussion, but the subject far exceeds the scope of this article and will not be discussed. Complexation by humic substances has even been incorporated into thermodynamic speciation models used in different fields (e.g. MINTEQA2 [9], WHAM [10], ECOSAT [11], etc.) in an attempt to simultaneously take into account the complexation of trace elements by 'classical' l.m.m. (low molecular mass) ligands and humic macromolecules. However, in spite of the amount of work done, adequately describing trace element complexation by humics has been, and essentially remains, a controversial issue. This jeopardises any possibility of evaluating the role of humics in environmental, toxicological or industrial systems in practice. The implementation of quality assurance practices in humic complexation measurement has never been attempted. Although the study of trace element binding by humics has some aspects in common with 'classical' equilibrium constant determination [12], it possesses a number of characteristics, largely intrinsic to humics themselves, that makes the problem fundamentally different. For this reason, quality assessment procedures developed for 'classical' constants cannot be applied directly to humic data. In this article, the nature of humic substances and the implications for the study of trace element complexation will be analysed first, then an overview of the current situation will be provided, and finally a strategy for the application of quality assurance practices will be proposed.

\section{The special nature of humic substances}

The difficulties inherent in the treatment of trace element complexation by humic substances are linked to their nature and derive from two intrinsic characteristics of these substances: their operational definition and their chemical binding heterogeneity.

Humic substances are organic macromolecules that can only be obtained by chemical and physical fractionation from environmental waters, sediments and soils. Because of their nature, they can be identified from such samples and separated into groups of compounds with similar operationally defined physicochemical characteristics-which will always depend on the procedure applied-but they cannot be isolated from environmental samples in a pure state (i.e. consisting of a single molecular structure). Nor they can be synthesised in a laboratory by using normal organic chemistry synthesis techniques. The implications are profound. Even if standard procedures exist for the isolation of humic substances [2,3], using such procedures will always give substances with "average properties that are remarkably similar" [2] but they will never be "the same' as is the case for any common chemical compound. Rather, each time humic substances are isolated, they will consist not of identical molecules but rather of a mixture of chemical molecules which have similar average properties but could, in principle, all be different individually.

Humic substances contain a high variety of functional groups. The main groups have been characterised relatively well [2]. They include, in decreasing order of typical content: carboxyl, phenolic and alcoholic hydroxyl, quinone and ketonic carbonyl, nitrogen- and sulphurcontaining groups. The prevalence of carboxyl and phenolic hydroxyl groups is responsible for their net negative charge in natural waters and their behaviour as polyelectrolytic compounds. It is possible to estimate from the acid-base properties of reference fulvic and humic acids [13] that (1) a fulvic molecule contains on average $6.6 \mathrm{mmol} \mathrm{g}^{-1}$ of carboxyl groups, which corresponds to one carboxylic group per six carbon atoms, or one group per aromatic ring, if distributed evenly, whereas a humic molecule contains on average $4.8 \mathrm{mmol} \mathrm{g}^{-1}$, which corresponds to one carboxylic group per ten carbon atoms; (2) the average phenolic content group is $1.4 \mathrm{mmol} \mathrm{g}^{-1}$, this is one phenolic group per 30 carbon atoms for both humic and fulvic acids. Furthermore [14], (3) hydroxyl and carbonyl groups, put together, are as abundant as carboxyl groups $\left(5-7 \mathrm{mmol} \mathrm{g}^{-1}\right)$. Thus, an average fulvic acid molecule (molecular weight $2000 \mathrm{~g} \mathrm{~mol}^{-1}$ ) would have one carboxylic, hydroxyl or carbonyl group every three carbon atoms. The same considerations apply to humic acids, which are larger in size and have slightly different compositional features (fewer carboxyl groups) [2]. Although, strictly speaking, such molecules cannot be considered individually to have a high number and variety of potential binding groups, since humics consist of a mixture of a number of these similar (but not identical) molecules, the result is that they effectively have a wide variety of different binding sites which differ in their chemical composition and in their electronic environment.

The different functional groups present in humic substances encompass a large range of binding affinities, from S-containing soft sites to hard O-containing sites (see [15] for site definitions). Moreover, as mentioned above, the different sites are not present in equal amounts; there are far fewer soft sites than hard sites. Complexation of trace elements by humics will therefore depend on the element considered, its concentration, and the humic to metal ratio. The fact that the presence of hard and soft metals in natural waters and soils usually follows a similar trend (i.e. hard metals, such as $\mathrm{Ca}$ or $\mathrm{Mg}$ are present at much higher concentrations than soft metals such as $\mathrm{Hg}$ ) and the environmental implications of this have been discussed in detail in the past [16]. Although the existence of a large variety of 
binding sites in humic substances, and the implications for the description and quantification of metal binding by these substances, have been described by different authors (i.e. "polydisperse mixture character [17], "heterogeneous complexing agent" [18, 19], "metal concentration effect" [20]), the study of humic complexation has often been hampered by ignoring it.

\section{Experimental constraints}

Before discussing the problem of representing and interpreting experimental complexation data, it is necessary to briefly summarise the nature of directly accessible experimental variables and the way in which they can be obtained. The usual approach to trace element complexation by humic substances has been by using titrations, i.e. a measurement is taken using a mixture of the trace element and the humic substance, then increments of metal ion are added and the variation in the free metal is recorded. Titration-type data have sometimes been obtained by performing experiments in batch mode (i.e. by preparing solutions containing different concentrations of the metal and the ligand and measuring the free metal, which often requires prior physical separation of the free and complexed humic from the free metal). A detailed description of the techniques most often used (e.g. ISE potentiometry, voltammetry, fluorescence, dialysis etc.), as well as of their advantages and limitations when applied to the study of humic substances, can be found in [19]. This reference is more than 20 years old, and although some aspects would require some updating, it remains the best reference.

The experimental methods that can be used are obviously significantly constrained by the need to entirely 'titrate' the wide range of metal concentrations required to 'see' all humic complexing sites. The ideal technique needs to be sensitive enough to be able to 'titrate' the strongest sites and precise enough to be able to 'see' the weakest sites, where the complexed metal concentration (i.e. the difference between total and free metal concentrations) has to be calculated from two values that are very close when approaching saturation. In practice, for most metals, only a section of the entire underlying theoretical titration curve is measured, this section being a function of the analytical window of the technique used. In all cases, and as would be expected given the existence of many complexing sites with superposing degrees of saturation along the titration, the titration curves obtained are always shapeless (see references [21, 22] for a discussion).

It is important to note that not only the titration range but also the meaning of the titration data obtained might be a function of the technique employed: the majority of the techniques used are subject to methodological limitations, or they imply fundamental assumptions in the interpretation of the analytical signal. Different techniques can therefore give different results even when applied to the same range of metal concentrations. In particular, it has clearly been shown that any determination of the complexation capacity (i.e. saturation of all complexing sites by the metal) is highly dependent upon the method (Fig. 1). However, the most important discrepancy among published results derives from the titration of humics over different titration windows, thus accessing different fractions of the sites present. When these data have been interpreted by calculating mean complexation values, as has been often the case (see next section), the dispersion of the values obtained has been enormous. The extent of the problem is illustrated in Fig. 2 where a compilation of literature data for equilibrium quotients (mean values calculated by considering humics acting as a single ligand and forming 1:1 complexes only) for complexation of $\mathrm{Cu}(\mathrm{II})$ by aquatic fulvic compounds is shown as a function of $\mathrm{pH}$. Note the dispersion at some $\mathrm{pH}$ values exceeds six logarithmic units.

A significant problem with most of the humic complexation data published over the years lies in the fact that

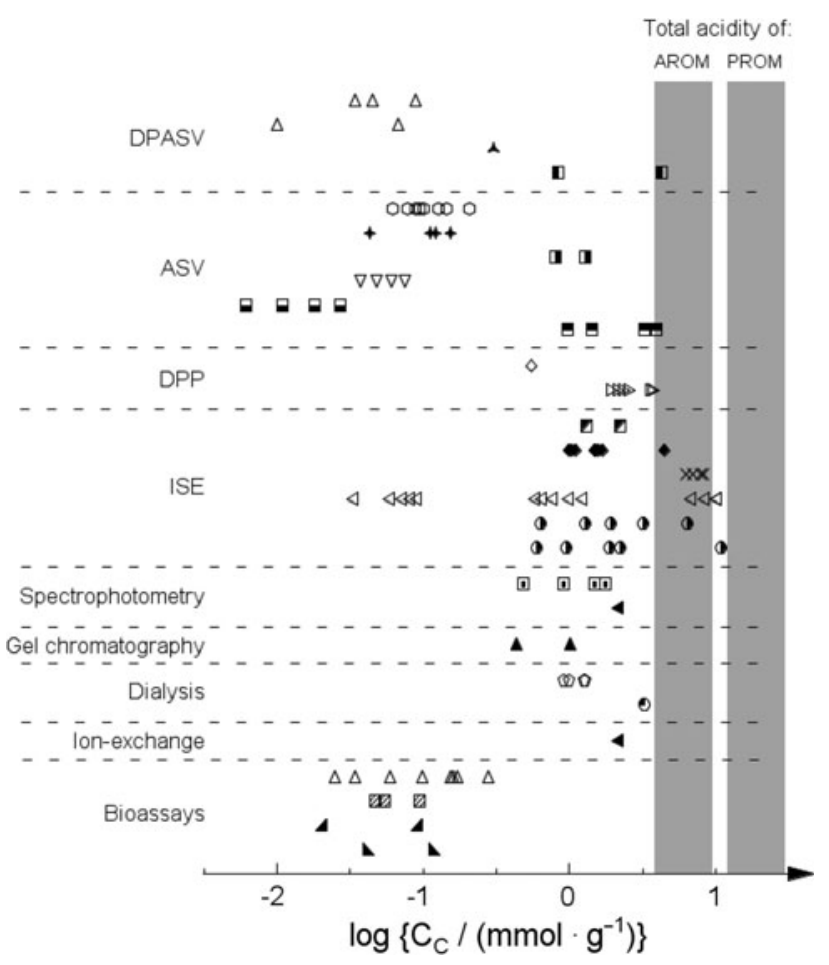

Fig. 1 Collection of published complexation capacity values, $C_{\mathrm{c}}$, the supposed maximum amount of complexing sites per carbon mass in $\left(\mathrm{mmol} \mathrm{g}^{-1}\right)$, for $\mathrm{Cu}(\mathrm{II})$-fulvic acid complexation. Each symbol corresponds to a different study. In most cases, $6<\mathrm{pH}<8$, $T=25{ }^{\circ} \mathrm{C}$, ionic strength $=0.1 \mathrm{~mol} \mathrm{~L}^{-1} . D P A S V$ differential pulse anodic stripping voltammetry, $A S V$ anodic stripping voltammetry, $D P P$ differential pulse polarography, ISE ionic selective electrode. $A R O M$ aquatic refractory organic matter, PROM soil refractory organic matter. Reproduced from [23] with permission (Elsevier) 


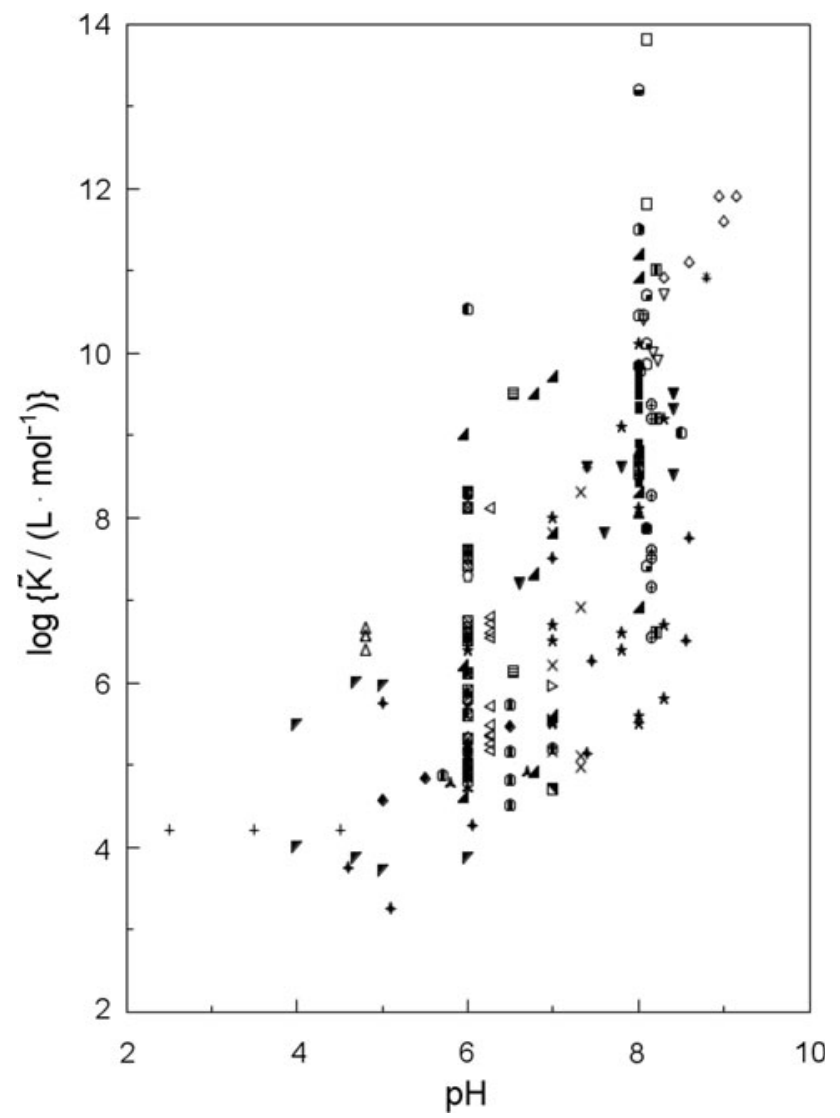

Fig. 2 Compilation of published values for $\mathrm{Cu}(\mathrm{II})$-water fulvic compounds complexation equilibrium quotients (1:1 stoichiometry), $\log \left\{\tilde{K} /\left(\mathrm{L} \mathrm{mol}^{-1}\right)\right\}$ as a function of $\mathrm{pH}$. Each symbol corresponds to a different study. Reproduced from [16] with permission (WileyBlackwell Publishing)

very high ligand and metal concentrations have been used, mainly because most available techniques were not sensitive enough, but also because most authors were not aware that the nature of humic substances makes it impossible to characterise trace metal complexation by humics in a meaningful way without taking into account the dependence of calculated constant values on the titrated metal concentration range. The result is that most published values of binding 'constants' only correspond to the complexation by the weakest sites (i.e. carboxylates), all of the strongest sites being saturated from the onset of the titration. This results in the 'constant' values being underestimated for many elements. Unfortunately, although the importance of this fact has been known for many years, many studies that entirely ignore it continue to be published.

Finally, although it will not be discussed in detail here, it is important to mention that, as is the case with 'classical' 1.m.m. ligands, published studies do not always contain high-quality experimental work and reporting is not always adequate. In particular, humic substances are not always well characterised and the reproducibility of the results is rarely tested, with 'constant' values often being calculated from a reduced number of titrations.

\section{The caveats of modelling}

The binding properties of any complexant vis-à-vis a metal ion in solution can, in principle, be described if the concentration of all binding sites, the stoichiometry of the complexes where they participate and the corresponding free energy (or equilibrium constant) of the equilibria are (or can be) known. However, in the case of humics, (1) the number and type of sites change depending on the experimental conditions (see previous section), (2) the total concentration of the humic substance present can only be expressed as mass per unit volume, but not in the molar scale, (3) titration curves - the only accessible experimental information-are the result of many different sites which are at different degrees of saturation and cannot be deconvoluted into the contributions of each site. This has led to different models being developed to describe binding. They mainly differ in how they tackle the problem of defining the 'ligand'. For instance, discrete models assume the existence of a limited number of sites, associated 'ligand' concentrations and equilibrium quotients; they range from the simplest model that considers the existence of only one site type (as in the case of values shown in Fig. 2) to a model which is quite popular nowadays (WHAM [10]) that includes a total of 476 possible sites, that, after some simplification, reduce to 80 sites [4]. On the other hand, continuous models are based on the assumption that humics incorporate a very large (almost infinite) number of different types of complexing sites and that site-type distinction can only be based on differences in the free energy of the complexation reaction, with complexation of a metal by humics being described by a continuous probability distribution of binding energies. It is beyond the scope of this article to provide a detailed description of, or even to list, the existing models. Review papers on the topic do exist, though unfortunately some of them cannot be considered particularly useful either because they just reflect the opinion of the author of one of the models, which inevitably is biased in favour of it, or they just contain a compilation of models without any further analysis.

All current humic models should be considered empirical models that do not provide a 'classical' thermodynamic description of binding [24]. All are based on a number of a priori chemical or mathematical assumptions (e.g. number of sites, binding isotherms, etc.). Not surprisingly, the more 'successful' models in terms of fitting experimental data contain a higher number of adjustable parameters. Moreover, to be applicable to systems other than the ones used for their 
parameterisation, they often require a further 'tuning' process (i.e. "the manipulation of the independent variables to obtain a match between the observed and simulated distribution or distributions of a dependent variable or variables" [25]), the concentration of complexing humic being the variable typically adjusted [26]. Thus, as pointed out by Nordstrom, "testing the models with laboratory or field data to assess their 'correctness' or 'validity' really tests their consistency with data, not necessarily their proximity to truth" [27]. Therefore, regardless of their usefulness in solving a particular problem, existing models can, in the best case scenario, only be described as "empirically adequate" [25].

\section{The current situation}

In the light of the contents of previous sections, the current situation, described as 'controversial' in the introduction, can be summarised as follows:

1. As a result of more than 50 years of data measuring, a relatively high number of studies that contain binding data for humics exist. However, these data have been obtained for humic substances which have been isolated by using different fractionation techniques, not always adequately characterised, under different conditions, for a wide variety of titration windows and often only for high concentrations. Different analytical techniques and interpretation models have been applied.

2. Data do not exist for most of the elements of the periodic table because most of the existing studies have been devoted to just a few elements.

3. No comprehensive compilation of published data exists. Attempts at data compilation have usually been guided by the need to supply particular models (e.g. $[28,29])$

4. There are no well-established methods for assessing the quality of existing data and for comparing data from different studies, unless exactly the same model and experimental conditions have been used, which essentially means that most of the published data can never be used.

5. The use of speciation codes embedding humic binding models that imply a high degree of fitting [26] is increasingly observed in some fields (e.g. bioavailability studies [30]).

\section{Data normalisation, an essential step}

The many and varied a priori assumptions contained in existing humic binding models preclude any direct comparison between published parameters resulting from different models. As such, it is essential to establish data quality assessment procedures that are independent of the model used to interpret the data. This requires, as a first step, the normalisation of raw binding data in a way that allows data to be compared, irrespective of the model eventually used by the authors to treat the experimental data. Two data normalisation procedures suggested in the literature will be described briefly below as an illustration but probably others exist or can be developed.

Metal loading versus free metal 'working curves'

The metal loading or 'degree of site occupation' at each titration point is defined as the quotient of the metal bound (mol L ${ }^{-1}$ ) at the titration point, calculated as the difference between two measured parameters (total and free metal ion concentrations), and the humic concentration expressed in $\mathrm{g} \mathrm{L}^{-1}$. This parameter's units will therefore be mol $\mathrm{g}^{-1}$ of humic substance (or mol $\mathrm{g}^{-1}$ of organic carbon if, as is often the case, the concentration of humics is expressed as organic carbon concentration). Metal loading is represented as a function of the free metal ion concentration $\left(\mathrm{mol} \mathrm{L}^{-1}\right)$. The choice of the free metal ion concentration as the independent variable is justified on the grounds that, although strictly speaking the free metal ion is a dependent variable, it can be taken as a master variable "reflecting global complexation energy (i.e. an intensive variable)" [31]. This type of normalisation therefore represents an extensive versus an intensive parameter. It has been shown that the slope of these curves is related to the metal buffer complexation capacity of the system [32].

This type of data normalisation was suggested by Buffle and co-workers more than 20 years ago [18, 19, 21, 23]. Different symbols have been used by these authors to represent the dependent variable, which may lead to some confusion for the users: $\bar{\theta}$ [18], $\bar{v}^{*}$ [19], $\theta[23],[M]_{\mathrm{b}} /\{S\}_{\mathrm{t}}$ [31] with $[M]_{\mathrm{b}}=c_{\mathrm{Mb}}=$ metal bound in $\mathrm{mol} \mathrm{L}^{-1}$ and $\{S\}_{\mathrm{t}}=\gamma_{\mathrm{t}}=$ concentration of humics in $\mathrm{g} \mathrm{L}^{-1}$. Other authors have used different symbols. For instance, Tipping used $v$ to denote moles of an element bound per gram of humic substances [4, 28], while Wageningen's research group used [Metal ads] with units mol kg ${ }^{-1}$ [33]. This type of data representation corresponds to the so-called 'isotherms' often used by soil scientists.

${ }^{\mathrm{c}} K$ versus free metal curves

Another approach to data normalisation [24, 29] combines titration data in a parameter ${ }^{\mathrm{c}} K$ defined as

${ }^{\mathrm{c}} K=\frac{c_{\mathrm{Mb}}}{c_{\mathrm{M}} \gamma_{\mathrm{t}}}$ 
where $c_{\mathrm{M}}=$ free metal ion concentration in $\mathrm{mol} \mathrm{L}^{-1}$. Note that $\gamma_{\mathrm{t}}$ is the mass concentration of humics in $\mathrm{g} \mathrm{L}^{-1}$ and should not be confused with an activity coefficient. The unit of ${ }^{\mathrm{c}} K$ is volume per mass, usually given as $\mathrm{L} \mathrm{g}^{-1}$. The parameter ${ }^{c} K$ has the same mathematical structure as the well-known equilibrium constant

$K=\frac{c_{\mathrm{ML}}}{c_{\mathrm{M}} c_{\mathrm{L}}}$

of metal-ligand complex formation for the reaction $\mathrm{M}+$ $\mathrm{L} \leftrightarrow \mathrm{ML}$. For scientists trained to think in terms of equilibrium constants, the ${ }^{\mathrm{c}} K$ versus free metal curves might therefore be more intuitive to work with than metal loading curves. However, this similitude may also be misleading and it is very important bear in mind the fact that, in spite of its form, ${ }^{\mathrm{c}} \mathrm{K}$ is not a 'classical' equilibrium constant but an empirically defined parameter. The relationships of ${ }^{\mathrm{c}} K$ and 'classical' equilibrium constants for different model assumptions have been discussed at length in reference [24].

In fact, both normalisation approaches are linked since ${ }^{\mathrm{c}} K$ can be obtained by dividing the metal loading parameter by the free metal ion concentration. Both types of normalisation curve are shown in Figs. 3 and 4 for the complexation of $\mathrm{Cu}$ by a purified peat humic acid at different $\mathrm{pH}$ values and of some lanthanides $(\mathrm{Eu}, \mathrm{Tb}, \mathrm{Dy})$ and trivalent actinides $(\mathrm{Am}, \mathrm{Cm})$ by different humic and fulvic acids, respectively.

One of the advantages of the metal loading normalisation approach is that it allows the direct comparison of the intensity of binding in comparable conditions, i.e. at the same metal loading. For instance, Fig. 3a clearly shows that, as expected, complexation is stronger at higher $\mathrm{pH}$ values (i.e. at the same metal loading value, free metal ion concentrations are lower at higher $\mathrm{pH}$ values). Data in Fig. 4a are interesting from a quality assessment point of view because they exemplify some of the common problems found in 'humic' studies. For instance, this figure shows that only one study [37] has been carried out over a reasonable metal ligand concentration range, with the two humic compounds studied showing indistinguishable complexation behaviour vis-à-vis Eu within the experimental variability observed. All other studies used concentrations which were too close to the saturation conditions for any conclusion to be drawn regarding the type of element or complexant, with the major differences observed (e.g. two studies for Eu and the same humic substance $[34,35]$ show, at the same metal loading conditions, differences in free metal ion concentration of up to two orders of magnitude) probably being linked to the measuring techniques used (problem already shown in Fig. 1).

Possible differences in binding properties at very low metal to ligand ratios are easy to observe in ${ }^{\mathrm{c}} K$ versus free
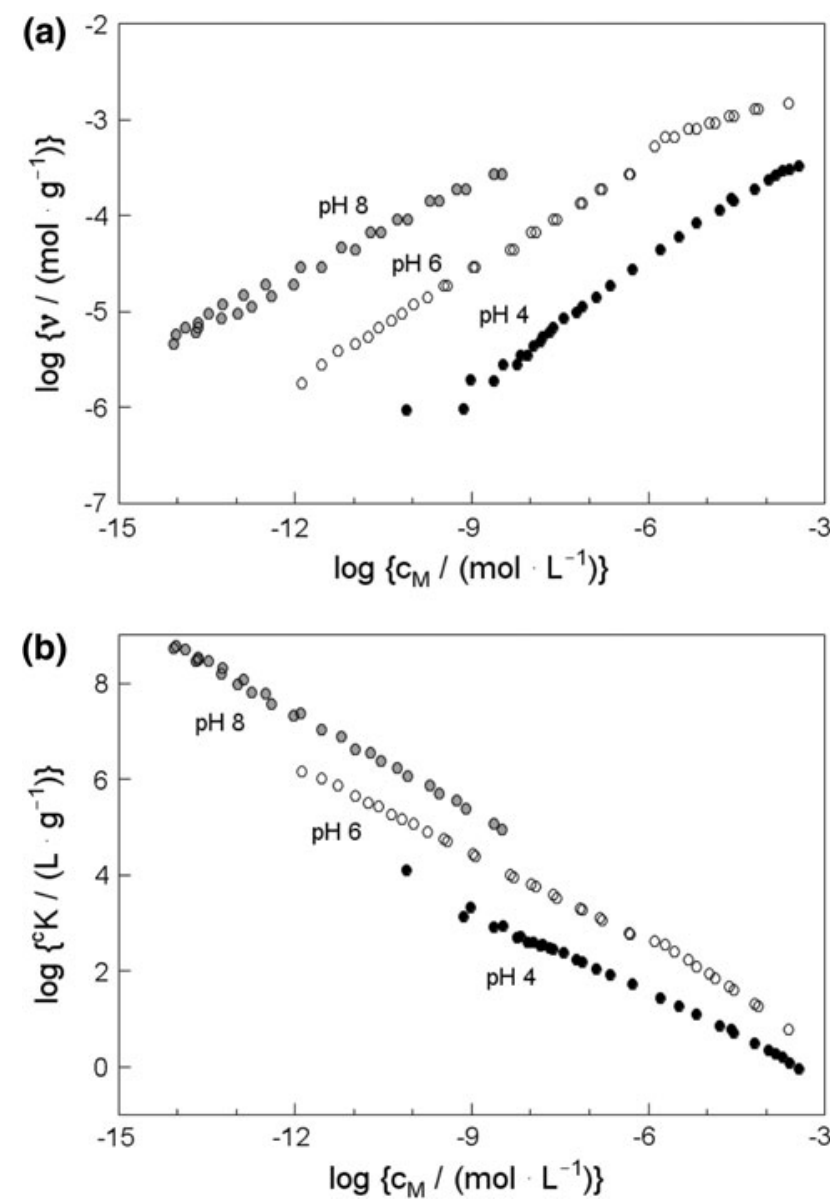

Fig. 3 Complexation of copper by a purified peat humic acid at different $\mathrm{pH}$ values [33]. a Metal loading, $\log \left\{\left(\mathrm{v} /\left(\mathrm{mol} \mathrm{g}^{-1}\right)\right\}\right.$, versus free metal ion concentration curves. $\mathbf{b} \log \left\{{ }^{\mathrm{c}} K /\left(\mathrm{L} \mathrm{g}^{-1}\right)\right\}$ versus free metal ion concentration curves

metal curves (Figs. 3b, 4b). When experimental windows extend to the region of the strongest binding sites (i.e. at very low metal loading), working curves become horizontal. In the cases shown, there is no curvature in the $\mathrm{Cu}$ data (Fig. 3b), whereas a slight curvature appears in some Eu curves (Fig. 4b). On the other hand, within the limits imposed by the scatter of the data points, a consistent overall pattern emerges for humic complexation with trivalent lanthanides and actinides (Fig. 4b).

\section{Uncertainties}

Normalised data should always be accompanied by an indication of their quality. The uncertainties associated with the parameters represented in the normalised curves can be evaluated according to well-established procedures [41], as long as the necessary information is provided by the authors. A more difficult problem is detecting systematic errors in systems such as the ones considered here 

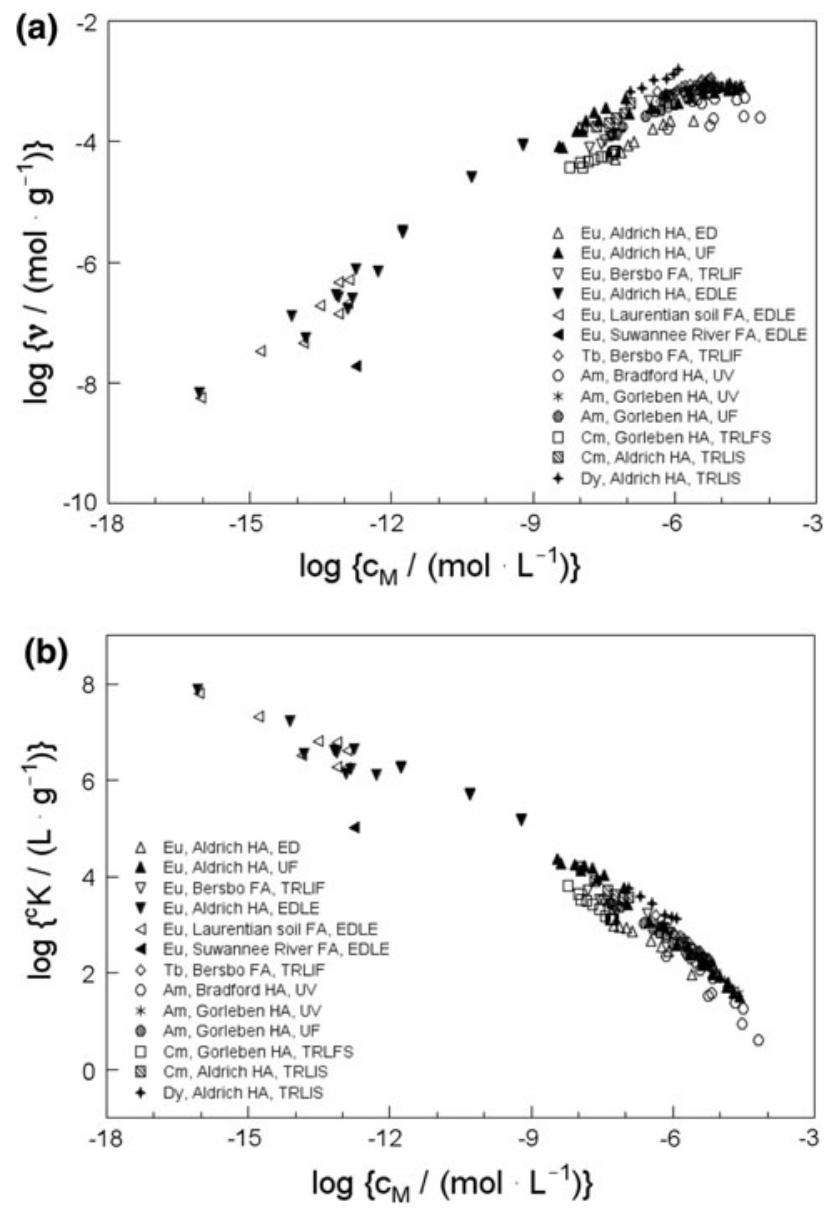

Fig. 4 Complexation of some lanthanides (Eu, Tb, Dy) and trivalent actinides $(\mathrm{Am}, \mathrm{Cm})$ by different humic and fulvic acids. a Metal loading $\log \left\{\left(v /\left(\mathrm{mol} \mathrm{g}^{-1}\right)\right\}\right.$ versus free metal ion concentration curves. b $\log \left\{{ }^{\mathrm{C}} K /\left(\mathrm{L} \mathrm{g}^{-1}\right)\right\}$ versus free metal ion concentration curves. Source: Eu, Aldrich HA, ED [34]; Eu Aldrich HA, UF [35]; Eu and Tb, Bersbo FA, TRLIF [36]; Eu, Aldrich HA, Laurentian soil FA and Suwannee River FA, EDLE [37]; Am, Bradford HA, UV [38]; Am and $\mathrm{Cm}$, Gorleben HA, UV, TRLFS and UF [39]; $\mathrm{Cm}$ and Dy, Aldrich HA, TRLIS [40]. Meaning of abbreviations: $E D$ equilibrium dialysis, $E D L E$ equilibrium dialysis with ligand exchange, $F A$ fulvic acid, HA humic acid, TRLFS time-resolved laser fluorescence spectroscopy, TRLIF time-resolved laser-induced fluorescence, TRLIS time-resolved laser-induced spectrofluorometry, $U F$ ultrafiltration, $U V \mathrm{UV} /$ vis spectroscopy

which contain a numerous sources of variability. However, comparing data normalised as described above provides a very efficient tool for the detection of systematic errors because the normalisation procedures eliminate the first source of variability (i.e. the dependence of binding intensity on metal loading) and its confounding effect when comparing mean binding parameters.

It would be extremely useful to identify some reference systems to be used prior to any complexation study in order to test the experimental approach used. A similar strategy was suggested years ago in 'classical' equilibrium constant determinations [42]. A current IUPAC project dealing with the compilation and analysis of all published data [6] may make an important contribution to this area.

\section{Suggested procedure}

The characterisation and quantification of trace element binding by humic substances is a particularly difficult subject from many perspectives. Most of the difficulties derive from the nature of the substances themselves which have intrinsic characteristics that do not fit well with the concepts and techniques familiar to chemists. Notably, particular features of humics make it necessary to apply normalisation procedures to the raw experimental data prior to any treatment (model) or application. They also impose specific demands on the experimental work and reporting practices. The following procedure is recommended:

- Data collection: the practices usually recommended for the determination of equilibrium constants for welldefined l.m.m. ligands apply (e.g. purity of the reagents, adequate characterisation of the ligand, use of independent solutions, control of temperature and ionic strength, checking of repeatability, etc.). In addition, two important aspects need to be considered: adequate interpretation of the analytical signal in the presence of humics and coverage of a large range of metal and ligand concentrations (which should include low metal concentrations in the case of most environmental applications). The evaluation of already published data also needs to consider all of these aspects.

- Data reporting: The usual recommendations apply [43, 44]. In addition, the experimental raw data (i.e. titrations) should always be included, preferably in the form of tables, either in the paper itself or as supplementary material associated with the publication. The experimental raw data have to include the concentrations of total and free trace element $\left(\mathrm{mol} \mathrm{L}^{-1}\right)$ and humics (in $\mathrm{g} \mathrm{L}^{-1}$ of humic substance or of organic carbon) in specified experimental conditions (temperature, $\mathrm{pH}$, ionic strength, electrolyte medium). If other types of raw experimental data are published, the information provided in the paper should be sufficient to allow these data to be transformed into the chosen normalisation parameters without any ambiguity. Data reporting should always include estimations of the uncertainty associated with the measurements.

- Data normalisation: A normalisation procedure should be applied to the raw data. This procedure will reveal any systematic errors (e.g. linked to the experimental method used) and will make it possible to estimate the uncertainty associated with the normalised data and identify the main variability factors characteristic of the 
system (e.g. type of metal, type of humic substance, $\mathrm{pH}$, etc.).

The introduction pointed out that trace element complexation by humics was a controversial issue. Implementing the above procedure will not completely solve the problem. However, irrespective of the difficulties involved, without a sound quality assessment of existing published data and new data, a way through the current deadlock will not be found.

\section{References}

1. Filella M (2007) Colloidal properties of submicron particles in natural waters. In: Wilkinson KW, Lead J (eds) Environmental colloids and particles. Behaviour, separation and characterisation, IUPAC-Wiley, pp 17-93

2. http://ihss.gatech.edu/ihss $2 /$. Last accessed 18 Oct 2010

3. Filella M (2009) Freshwaters: which NOM matters? Environ Chem Lett 7:21-35

4. Tipping E (2002) Cation binding by humic substances. Cambridge University Press, Cambridge

5. Koopal LK, Saito T, Pinheiro JP, van Riemsdijk WH (2005) Ion binding to natural organic matter: general considerations and the NICA-Donnan model. Coll Surf A 265:40-54

6. Humic-Metal Binding Constants Database, IUPAC project 2008025-1-500

7. Coleman NT, McClung AC, Moore DP (1956) Formation constants for $\mathrm{Cu}(\mathrm{II})$-peat complexes. Science 123:330-331

8. Himes FL, Barber SA (1957) Chelating ability of soil organic matter. Soil Sci Soc Am Proc 21:368-373

9. http://www.epa.gov/ceampubl/mmedia/minteq/. Last accessed 18 Oct 2010

10. http://windermere.ceh.ac.uk/aquatic_processes/wham/index.html. Last accessed 18 Oct 2010

11. http://www.soq.wur.nl/UK/Research/ECOSAT/. Last accessed 18 Oct 2010

12. Filella M, May PM (2010) Chemical modelling of multicomponent mixtures: why quality assurance encompasses more than pure equilibrium data quality assessment and how it can be achieved. Accred Qual Assur. doi:10.1007/s00769-010-0701-x

13. Ritchie JD, Perdue EM (2003) Proton-binding study of standard and reference fulvic acids, humic acids, and natural organic matter. Geochim Cosmochim Acta 67:85-96

14. Thurman EM (1985) Organic geochemistry of natural waters. Martinus Nijhoff/Dr W. Junk Publishers, Dordrecht

15. Duffus JF (2002) "Heavy metals"-a meaningless term? Pure Appl Chem 74:793-807

16. Filella M, Town RM, Buffle J (2002) Speciation in freshwaters. In: Ure AM, Davidson CM (eds) Chemical speciation in the environment, 2nd edn. Blackwell, Oxford, pp 188-236

17. Gamble DS, Underdown AW, Langford CH (1980) Copper(II) titration of fulvic acid ligand sites with theoretical, potentiometric, and spectrophotometric analysis. Anal Chem 52:1901-1908

18. Buffle J, Altmann RS (1987) Interpretation of metal complexation by heterogeneous complexants. In: Stumm W (ed) Aquatic surface chemistry: chemical processes at the particle-water interface. Wiley, New York, pp 351-383

19. Buffle $\mathbf{J}$ (1988) Complexation reactions in aquatic systems. An analytical approach. Ellis Horwood, Chichester

20. Hummel W, Glaus MA, Van Loon LR (1999) Complexation of radionuclides with humic substance: the metal concentration effect. Radiochim Acta 84:111-114
21. Altmann RS, Buffle J (1988) The use of differential equilibrium functions for interpretation of metal binding in complex ligand systems: its relation to site occupation and site affinity distributions. Geochim Cosmochim Acta 52:1505-1519

22. Filella M (2008) NOM site binding heterogeneity in natural waters: discrete approaches. J Mol Liquids 143:42-51

23. Buffle J, Filella M (1995) Physico-chemical heterogeneity of natural complexants: clarification. Anal Chim Acta 313:144-150

24. Hummel W (1997) Binding models for humic substances. In: Grenthe I, Puigdomenech I (eds) Modelling in aquatic chemistry. Nuclear Energy Agency, Paris, pp 153-206

25. Oreskes N, Shrader-Frechette K, Belitz K (1994) Verification, validation, and confirmation of numerical models in the Earth Sciences. Science 263:641-645

26. Filella M (2010) Quantifying 'humics' in freshwaters: purpose and methods. Chem Ecol 26:177-186

27. Nordstrom DK (1993) EOS Trans Am Geophys Union Suppl April 20326

28. Tipping E, Hurley MA (1992) A unifying model of cation binding by humic substances. Geochim Cosmochim Acta $56: 3627-3641$

29. Hummel W, Glaus MA, Van Loon LR (2000) Trace metalhumate interactions. II. The "conservative roof" model and its application. Appl Geochem 15:975-1001

30. www.epa.gov/waterscience/standards/academy/special/blm/index. html. Last accessed 18 Oct 2010

31. Buffle J, Altmann RS, Filella M, Tessier A (1990) Complexation by natural heterogeneous compounds: site occupation distribution functions, a normalized description of metal complexation. Geochim Cosmochim Acta 54:1535-1553

32. Buffle J, Altmann RS, Filella M (1990) The effect of physicochemical heterogeneity of natural complexants. II. The buffering action and role of their background sites. Anal Chim Acta 232:225-237

33. Benedetti MF, Milne CJ, Kinniburgh DG, van Riemsdijk WH, Koopal LK (1995) Metal ion binding to humic substances: application of the non-ideal competitive adsorption model. Environ Sci Technol 29:446-457

34. Carlsen L, Bo P, Larsen G (1984) Radionuclide-humic acid interactions studied by dialysis. In: Barney GS, Navratil JD, Schulz WW (eds) Geochemical behavior of disposed radioactive waste. American Chemical Society, Washington, DC, pp 167-180

35. Caceci MS (1985) The interaction of humic acid with europium(III). Complexation strength as a function of load and $\mathrm{pH}$. Radiochim Acta 39:51-56

36. Bidoglio G, Grenthe I, Qi P, Robouch P, Omenetto N (1991) Complexation of europium and terbium with fulvic acids as studied by time-resolved laser-induced fluorescence. Talanta 38:999-1008

37. Glaus MA, Hummel W, Van Loon LR (1997) Experimental determination and modeling of trace metal-humate interactions: a pragmatic approach for applications in groundwater. PSI Report No. 97-13, Paul Scherrer Institute, Villigen, Switzerland. Also published as Nagra Technical Report NTB 97-03, Nagra, Wettingen, Switzerland

38. Kim JI, Buckau G, Bryant E, Klenze R (1989) Complexation of americium(III) with humic acid. Radiochim Acta 48:135-143

39. Kim JI, Rhee DS, Wimmer H, Buckau G, Klenze R (1993) Complexation of trivalent actinide ions $\left(\mathrm{Am}^{3+}, \mathrm{Cm}^{3+}\right)$ with humic acid: a comparison of different experimental methods. Radiochim Acta 62:35-43

40. Moulin V, Tits J, Moulin C, Decambox P, Mauchien P, de Ruty O (1992) Complexation behaviour of humic substances towards actinides and lanthanides studied by time-resolved laser-induced spectrofluorometry. Radiochim Acta 58/59:121-128 
41. Ellison SLR, Rosslein M, Williams A (eds) (2000) Quantifying uncertainty in analytical measurement. 2 edn. EURACHEM/CITAC Guide CG 4

42. Braibanti A, Ostacoli G, Paoletti P, Pettit LD, Sammartano S (1987) Recommended procedure for testing the potentiometric apparatus and technique for the $\mathrm{pH}$-metric measurement of metalcomplex equilibrium constants. Pure Appl Chem 59:1721-1728
43. Tuck DG (1989) A proposal for the use of a standard format for the publication of stability constant measurements. Pure Appl Chem 61:1161-1163

44. Filella M, May PM (2005) Reflections on the calculation and publication of potentiometrically determined formation constants. Talanta 65:1221-1225 